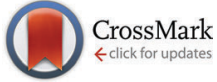

Cite this: Chem. Commun., 2015, 51, 9385

Received 13th March 2015, Accepted 30th April 2015

DOI: $10.1039 / \mathrm{c} 5 \mathrm{cc0} 02170 \mathrm{~g}$

www.rsc.org/chemcomm

\section{VCD spectroscopy as an excellent probe of chiral metal complexes containing a carbon monoxide vibrational chromophore $\uparrow$}

\author{
Marco Fusè $\ddagger^{\mathrm{a}}$ Giuseppe Mazzeo, $\ddagger^{\mathrm{b}}$ Giovanna Longhi, ${ }^{\mathrm{bc}}$ Sergio Abbate, ${ }^{\mathrm{bc}}$ \\ Daniele Zerla, ${ }^{a}$ Isabella Rimoldi, ${ }^{a}$ Alessandro Contini ${ }^{a}$ and Edoardo Cesarotti*a
}

\begin{abstract}
Vibrational circular dichroism, VCD, gives evidence that the carbon monoxide chromophore in a heteroleptic cyclopentadienyl Ru(II)carbonyl complex is very sensitive to the chirality of the metal centre and becomes an excellent probe to define the configuration of chiral metal complexes.
\end{abstract}

Carbon monoxide is a common ligand for transition metals and very frequently appears in coordination complexes: metal carbonyls act as catalysts or catalyst precursors in a great variety of catalytic and stoichiometric reactions. Most often $\mathrm{CO}$ occupies one coordination site acting as a linearly coordinated ligand.

In mononuclear complexes the $\mathrm{CO}$ moiety rather strongly binds the transition metal via a $\sigma$ bond matched with a synergistic $\pi$ bond formed by the overlap of the $\pi^{*}$ of CO and the occupied d orbitals of the metal. The carbonyl HOMO has a partial antibonding character; thus the $\sigma$ bond electron density donation into the empty d orbital of the metal increases the stretching frequency which is lowered by the $\pi$ back-bond, increasing the electron density in the antibonding CO $\pi^{*}$ orbital. This assertion is particularly true for the late transition metals in a low oxidation state where the $\pi$ back-bond effect exceeds the $\sigma$ bond one. The $\nu$-CO stretching vibration has a value of $2143 \mathrm{~cm}^{-1}$, typical of free CO as recorded in the gas phase. ${ }^{1}$ In classical mononuclear carbonyl complexes, it lies in the range $2125-1850 \mathrm{~cm}^{-1}, 2$ a region of the IR spectrum devoid of other signals; the corresponding absorption bands are sharp with medium to high intensity. Therefore, infra-red (IR) spectroscopy is known to be a valuable technique to study carbonyl complexes and their electronic properties. In heteroleptic carbonyl

\footnotetext{
${ }^{a}$ Dipartimento di Scienze Farmaceutiche, Università di Milano Via Golgi 19, 20133 Milano, Italy. E-mail: edoardo.cesarotti@unimi.it

${ }^{b}$ Dipartimento di Medicina Molecolare e Traslazionale, Università di Brescia, Viale Europa 11, 25123 Brescia, Italy

${ }^{c}$ CNISM Consorzio Nazionale Interuniversitario per le Scienze Fisiche della Materia,

Via della Vasca Navale, 84, 00146 Roma, Italy

$\dagger$ Electronic supplementary information (ESI) available. See DOI: 10.1039/ c5cc02170g

\$ These authors equally contributed to this work.
}

complexes bearing a chiral metal centre, vibrational circular dichroism (VCD), i.e. CD in the infra-red region, ${ }^{3-11}$ is expected to be particularly adequate to study metal complexes. In particular the VCD signal of the stretching vibration promises to be quite useful to investigate the chirality at the metal centre and may become an analytical technique to assign the configuration of chiral transition metals. VCD has already been used ${ }^{12-14}$ to study metal complexes but never to investigate the linear coordinated carbon monoxide as a probe of chirality at the metal centre.

Since the pioneering studies of Brunner et al. ${ }^{15-18}$ a number of chiral carbonyl complexes are known for which the unambiguous assignment of the configuration is based on their X-ray crystal structure. In this work we report the VCD investigation in solution for a couple of carbonyl complexes among the first ones isolated in an optically pure form, T-4- $R-\eta 5-((1 R, 2 S, 5 R)-$ menthyl $)-\mathrm{C}_{5} \mathrm{H}_{4} \mathrm{Ru}(\mathrm{CO})\left(\mathrm{P}\left(\mathrm{C}_{6} \mathrm{H}_{5}\right)_{3}\right) \mathrm{Cl}\left((R)_{\mathrm{Ru}}\right)$ and $\mathrm{T}-4-S-\eta 5-((1 R, 2 S, 5 R)-$ menthyl $)-\mathrm{C}_{5} \mathrm{H}_{4} \mathrm{Ru}(\mathrm{CO})\left(\mathrm{P}\left(\mathrm{C}_{6} \mathrm{H}_{5}\right)_{3}\right) \mathrm{Cl}\left((S)_{\mathrm{Ru}}\right),{ }^{19}$ together with a complete NMR characterization and density functional theory (DFT) calculations. ${ }^{3,5,6,11}$

$(R)_{\mathrm{Ru}}$ and $(S)_{\mathrm{Ru}}{ }^{20}$ are stable half sandwich, three-legged piano stool cyclopentadienyl complex in which the ruthenium atom is in a slightly distorted octahedral geometry; due to the three cis positions occupied by the $\eta 5$-cp ring, the diastereomers can be defined according to the extended CIP sequence: ${ }^{21} \eta 5-((1 R, 2 S, 5 R)-$ menthyl) $-\mathrm{C}_{5} \mathrm{H}_{4}>\mathrm{Cl}>\mathrm{P}>\mathrm{CO}$ (Fig. 1).

$(R)_{\mathrm{Ru}}$ and $(S)_{\mathrm{Ru}}$ were prepared following a modified procedure of that reported in ref. 20 (refluxing heptane instead of xylene) and obtained as the pure diastereomers by preparative flash chromatography. The electronic circular dichroism (ECD) spectra of $(S)_{\mathrm{Ru}}$ showed an intense negative transition at $310 \mathrm{~nm}$ followed by a positive broad doublet in the range $350-500 \mathrm{~nm}$, similar to analogous neomenthyl complexes; ${ }^{22,23}(R)_{\mathrm{Ru}}$ showed nearly a mirror-image spectrum thus indicating that the ruthenium atoms are almost in an enantiomeric relationship at least in the ligand field transition range. It is interesting to note that the latter ECD features have the same sign as optical rotation (OR), $(-)$ for $(R)_{\mathrm{Ru}}$ and $(+)$ for $(S)_{\mathrm{Ru}}$. This is part of a general empirical rule derived from the Kramers-Kronig relationship between $\mathrm{OR}$ and $\mathrm{CD} .^{24,25}$ 

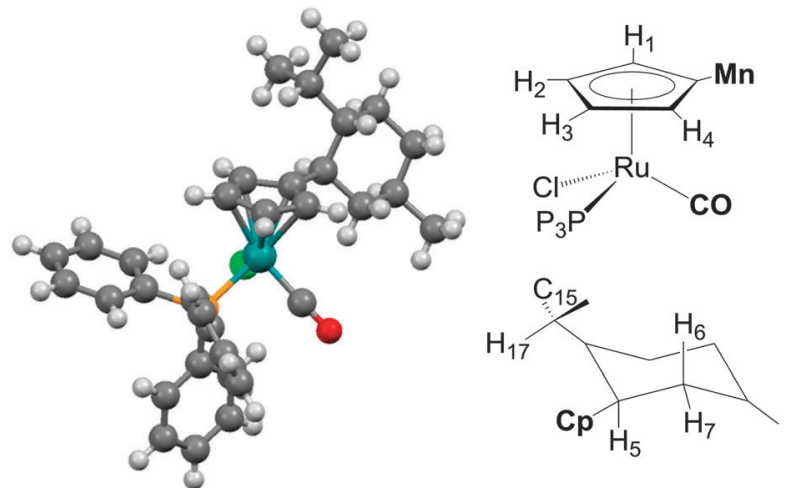

Fig. 1 Left: the molecular structures of $(S)_{R u}$ taken from ref. 20. Right: relevant proton labels on $\mathrm{Cp}$ (top) and menthyl (bottom) rings; the complete labels are reported in the ESI. $\dagger$

The NOESY spectra of both complexes revealed a very specific pattern: the hindered menthyl and $\mathrm{P}(\mathrm{Ph})_{3}$ moieties occupy an antipodal position. Moreover, the presence of cross-peaks of each proximal hydrogen on the $\mathrm{Cp}$ ring $\left(\mathrm{H}_{1}\right.$ and $\left.\mathrm{H}_{4}\right)$ with different portions of the menthyl moiety implies severe restriction of menthyl rotation or puckering, with the isopropyl fragment oriented farther from the metal, as highlighted by the presence of only one cross-peak between the aryl proton of $\mathrm{P}(\mathrm{Ph})_{3}$ and $\mathrm{H}_{7}$. In solution at $300 \mathrm{~K}$ it is remarkable that both diastereomers adopt a conformation with the bulky menthyl and $\mathrm{P}(\mathrm{Ph})_{3}$ groups as far away as possible from each other quite similarly as observed in the solid by X-ray spectroscopy on $(S)_{\mathrm{Ru}}$. In Table 1 are reported the distances determined by $\mathrm{NOE}^{26,27}$ together with the ones calculated by DFT and the ones determined by XRD for $(S)_{\mathrm{Ru}}$. The distances from XRD and NOESY are in excellent agreement.

In Fig. 2 we superimpose the experimental IR and VCD spectra of $(R)_{\mathrm{Ru}}$ and $(S)_{\mathrm{Ru}}$ : the monosignated VCD feature associated with CO stretching observed at $1955 \mathrm{~cm}^{-1}$ corresponding to the strong IR band inverts its sign in going from $(R)_{\mathrm{Ru}}$ to $(S)_{\mathrm{Ru}}$. Interestingly the sign of the CO-stretching VCD band is the same as that for OR and for the lowest energy ECD band.

Table 1 Atom-atom distances in $(S)_{\mathrm{Ru}}$ and $(R)_{\mathrm{Ru}}$ as evaluated from NOE spectra, X-ray diffraction data, and calculated DFT data of the structures which are in best agreement with X-ray and NOE data (the structures of the lowest energy found conformers are reported in the ESI)

\begin{tabular}{|c|c|c|c|c|c|}
\hline & \multicolumn{3}{|l|}{$\underline{(S)_{\mathrm{Ru}}}$} & \multicolumn{2}{|l|}{$(R)_{\mathrm{Ru}}$} \\
\hline & $\mathrm{XRD}^{a}$ & $\mathrm{NOE}^{b}$ & $\operatorname{DFT}^{c}$ & NOE & DFT \\
\hline $\mathrm{H}_{1}-\mathrm{H}_{5}$ & 2.69 & 2.8 & 2.6 & 2.8 & 2.7 \\
\hline $\mathrm{H}_{1}-\mathrm{H}_{17}$ & 2.92 & 2.8 & 3.1 & 2.7 & 2.9 \\
\hline $\mathrm{H}_{1}-\mathrm{C}_{15}$ & 2.77 & 2.9 & 2.6 & 2.8 & 2.5 \\
\hline $\mathrm{H}_{4}-\mathrm{H}_{6}$ & 2.41 & 2.5 & 2.4 & 2.4 & 2.3 \\
\hline $\mathrm{H}_{4}-\mathrm{H}_{7}$ & 3.21 & 3.7 & 3.3 & n.d. & 3.1 \\
\hline $\mathrm{H}_{1}-o \mathrm{Ph}^{d}$ & 3.18 & 3.1 & 2.4 & 2.9 & 2.6 \\
\hline $\mathrm{H}_{4}-O \mathrm{Ph}$ & 5.85 & 4.0 & 4.4 & 3.7 & 4.2 \\
\hline $\mathrm{H}_{2}-O \mathrm{Ph}$ & 2.80 & 2.9 & 2.8 & 2.8 & 2.6 \\
\hline $\mathrm{H}_{3}-O \mathrm{Ph}$ & 2.78 & 3.0 & 3.1 & 2.9 & 3.1 \\
\hline $\mathrm{H}_{7}-o \mathrm{Ph}$ & 4.30 & 4.6 & 4.3 & 4.2 & 4.5 \\
\hline
\end{tabular}

${ }^{a}$ Taken from ref. 20. ${ }^{b}$ This work, see ESI. ${ }^{c}$ This work, see ESI. ${ }^{d}$ Hydrogen at the ortho position in the phenyl ring of phosphine, see ESI.

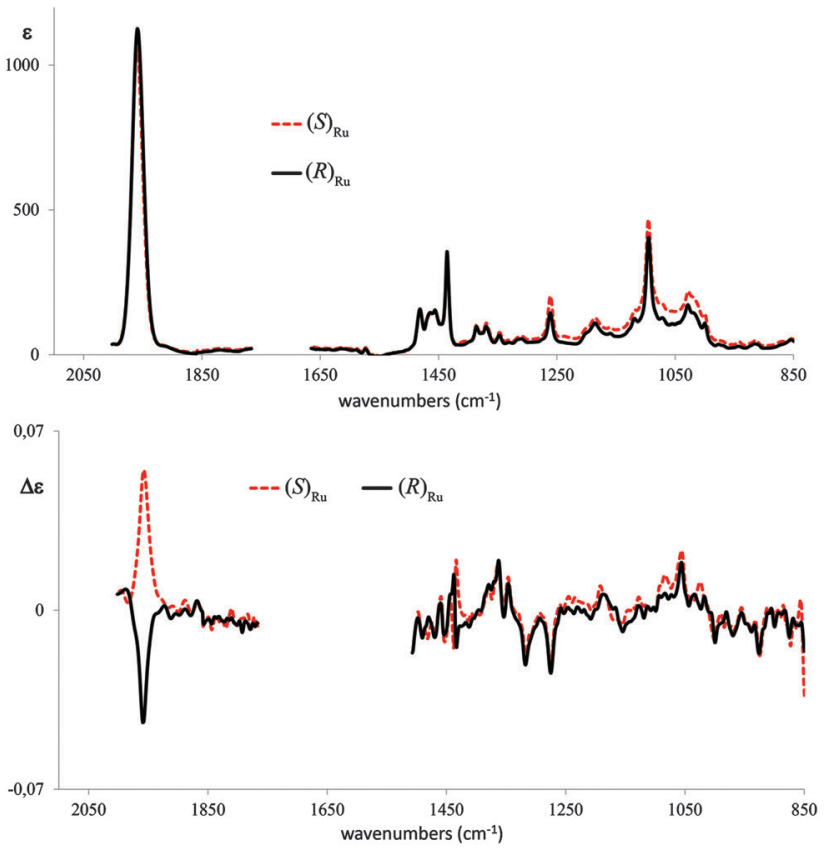

Fig. 2 IR absorption and VCD spectra of $(R)_{\mathrm{Ru}}$ and $(S)_{\mathrm{Ru}}$ complexes; in VCD spectra the band associated with $\mathrm{CO}$-stretching is directly related to Ru chirality, while the mid-IR VCD feature is mainly related to the menthyl moiety (see text).

In contrast, the $1700-850 \mathrm{~cm}^{-1}$ region exhibits several weaker VCD bands with the same sign for the two diastereomers, $(R)_{\mathrm{Ru}}$ and $(S)_{\mathrm{Ru}}$, with just two little exceptions: the couplet at $1420 \mathrm{~cm}^{-1}$ and the weak monosignated band at $c a .1000 \mathrm{~cm}^{-1}$. Based on this, we may attribute the VCD bands in the latter region to normal modes localized mainly in the menthyl moiety (and, to a lesser extent, in the cyclopentadienyl and phenyl moieties). Thus the sign of the corresponding VCD bands depends only on the configuration of the three asymmetric carbons in the menthyl, which does not change in going from $(R)_{\mathrm{Ru}}$ to $(S)_{\mathrm{Ru}}$ and bears good resemblance to the VCD spectra of menthone and menthol. ${ }^{28}$ Instead, the $1955 \mathrm{~cm}^{-1}$ band, associated with CO stretching, is directly related in sign to Ru chirality. DFT results presented below support our conclusion.

With regard to the intensities of VCD bands, the dissymmetry factor $g=(\Delta \varepsilon / \varepsilon)$ of the strongest VCD band, namely the CO stretching, is of the order of $10^{-4}$, which means that the intensity of the CO-stretching VCD band measured here is lower or comparable to other cases where coupled $\mathrm{C}=\mathrm{O}$ stretching gives rise to an exciton chirality effect ${ }^{29-31}$; however, it is quite intense when compared to the $\mathrm{VCD}$ of the isolated $\mathrm{C}=\mathrm{O}$ bond stretching in other molecules (e.g. for camphor ${ }^{11}$ ). Indeed our $\mathrm{Ru}(\mathrm{II})$ complexes are diamagnetic and not paramagnetic ${ }^{5}$ like the proline-cobalt complex ${ }^{3}$ where the intensification of VCD bands is due to the paramagnetic nature of $\mathrm{Co}(\mathrm{II})$. Confirmed also by the decrease of the VCD signal when $\mathrm{Co}$ (II) is oxidized to $\mathrm{Co}$ (III), it is worth mentioning that in those Co(II) complexes the carbonyl is bound to the metal by the oxygen and not by the carbon; however, our complexes resemble the situation of diamagnetic $\mathrm{Co}(\mathrm{III})$ but with the bonus that the Ru-CO VCD signal is directly connected to the 

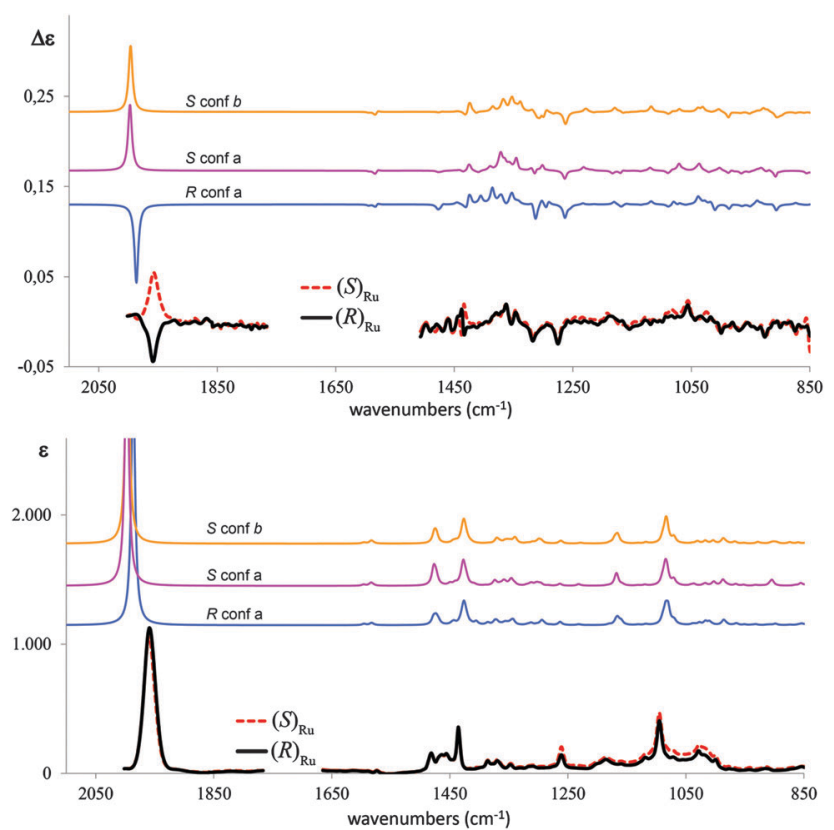

Fig. 3 Comparison of experimental VCD (top) and IR (bottom) spectra of $(R)_{\mathrm{Ru}}$ and $(S)_{\mathrm{Ru}}$ with corresponding calculated spectra for the conformers a and $\mathrm{b}$ of $(S)_{\mathrm{Ru}}$ and conformer a of $(R)_{\mathrm{Ru}}$ in Table 1, chosen on the basis of NOE distances (all conformers are characterized in Section 8, ESI $\dagger$ ).

stereochemistry of the metal and it becomes a good probe of Ru-chirality.

After a conformational search, DFT calculations were conducted at the $\mathrm{B} 97 \mathrm{D}^{32,33} / \mathrm{SVP}^{34}$ level. The geometrical data for the four structures with the lowest energy of both $(R)_{\mathrm{Ru}}$ and $(S)_{\mathrm{Ru}}$ are reported in Table 1 . These complexes allow for large amplitude hindered rotational modes. ${ }^{35,36}$ In order to establish the preferred conformation, we thus decided to compare the distances for the calculated structures with the experimental distances determined on the basis of NOE data for both $(R)_{\mathrm{Ru}}$ and $(S)_{\mathrm{Ru}}$ and X-ray diffraction data for $(S) \mathrm{Ru}^{20}$ As reported in Table 1 and Section 8 (ESI $\dagger$ ) the best compatibility between calculated and experimental distances is ensured by the conformers a and b for $(S)_{\mathrm{Ru}}$ and by conformer a for $(R)_{\mathrm{Ru}}$. The 3-D calculated structures are provided in the ESI. $\dagger$ In Fig. 3 we compare the experimental IR (lower) and VCD (higher) spectra for $(R)_{\mathrm{Ru}}$ and $(S)_{\mathrm{Ru}}$, with the calculated IR and VCD spectra of the conformers of the diastereomers $(R)_{\mathrm{Ru}}$ and $(S)_{\mathrm{Ru}}$, chosen as described above. While the mid-IR region is predicted to be independent of the configuration of the metal atom and shows weak signals, the CO stretching region inverts sign upon change in the configuration at the metal. A very good correspondence is found not only for the sign of the VCD bands but also for the IR and VCD intensities.

Through VCD spectroscopy we have given strong evidence that the carbon monoxide vibrational chromophore in a $\mathrm{Ru}(\mathrm{II})$ heteroleptic carbonyl complex is very sensitive to the chirality of the metal centre; with synthetic work in progress it will be possible to confirm the feasibility to use this new probe to determine the chirality of a metal centre.

\section{Notes and references}

1 G. Herzberg, Molecular Spectra and Molecular Structure, D. Van Nostrand Company Inc., 1950.

2 L. J. Bellamy, The infra-red spectra of complex molecules, Chapman and Hall London, 1975, vol. 1.

3 P. J. Stephens, F. J. Devlin, F. Gasparrini, A. Ciogli, D. Spinelli and B. Cosimelli, J. Org. Chem., 2007, 72, 4707-4715.

4 N. Berova, K. Nakanishi and R. Woody, Circular dichroism: principles and applications, John Wiley \& Sons, 2000.

5 P. L. Polavarapu and C. Zhao, Fresenius' J. Anal. Chem., 2000, 366, 727-734.

6 V. P. Nicu and E. J. Baerends, J. Phys. Chem. A, 2008, 112, 6978-6991.

7 S. Abbate, L. F. Burgi, F. Gangemi, R. Gangemi, F. Lebon, G. Longhi, V. M. Pultz and D. A. Lightner, J. Phys. Chem. A, 2009, 113, 11390-11405.

8 V. P. Nicu, E. Debie, W. Herrebout, B. Van der Veken, P. Bultinck and E. J. Baerends, Chirality, 2009, 21, E287-E297.

9 A. Bouchet, T. Brotin, M. Linares, H. Ågren, D. Cavagnat and T. Buffeteau, J. Org. Chem., 2011, 76, 1372-1383.

10 P. L. Polavarapu, J. Frelek and M. Woźnica, Tetrahedron: Asymmetry, 2011, 22, 1720-1724.

11 L. A. Nafie, Vibrational Optical Activity, John Wiley \& Sons, Ltd., 2011.

12 Y. He, X. Cao, L. A. Nafie and T. B. Freedman, J. Am. Chem. Soc., 2001, 123, 11320-11321.

13 H. Sato, Y. Mori, Y. Fukuda and A. Yamagishi, Inorg. Chem., 2009, 48, 4354-4361.

14 S. R. Domingos, A. Huerta-Viga, L. Baij, S. Amirjalayer, D. A. E. Dunnebier, A. J. C. Walters, M. Finger, L. A. Nafie, B. de Bruin, W. J. Buma and S. Woutersen, J. Am. Chem. Soc., 2014, 136, 3530-3535.

15 H. Brunner and J. Strutz, Z. Naturforsch., B: J. Chem. Sci., 1974, 29b, 446-447.

16 H. Brunner and E. Schmidt, J. Organomet. Chem., 1973, 50, 219-225.

17 H. Brunner, Angew. Chem., Int. Ed., 1999, 38, 1194-1208.

18 S. J. Cook, J. F. Costello, S. G. Davies and H. T. Kruk, J. Chem. Soc., Perkin Trans. 1, 1994, 2369-2372.

19 A. von Zelewsky, Stereochemistry of Coordination Compounds, John Wiley \& Sons, Ltd, 1996.

20 E. Cesarotti, G. Ciani and A. Sironi, J. Organomet. Chem., 1981, 216, 87-95.

21 R. S. Cahn, C. Ingold and V. Prelog, Angew. Chem., Int. Ed. Engl., 1966, 5, 385-415.

22 E. Cesarotti, M. Angoletta, N. P. C. Walker, M. B. Hursthouse, R. Vefghi, P. A. Schofield and C. White, J. Organomet. Chem., 1985, 286, 343-360.

23 E. Cesarotti, A. Chiesa, G. F. Ciani, A. Sironi, R. Vefghi and C. White, J. Chem. Soc., Dalton Trans., 1984, 653-661.

24 E. Giorgio, R. G. Viglione, R. Zanasi and C. Rosini, J. Am. Chem. Soc., 2004, 126, 12968-12976.

25 A. Moscowitz, Advances in Chemical Physics, John Wiley \& Sons, Inc., 2007, pp. 67-112.

26 L. Cronin, C. L. Higgitt and R. N. Perutz, Organometallics, 2000, 19, 672-683.

27 D. Neuhaus and M. P. Williamson, The Nuclear Overhauser Effect in Structural and Conformational Analysis, Wiley-VCH, 2000.

28 K. Konno, I. Shiina and H. Yui, J. Mol. Struct., 2013, 1035, 260-266.

29 S. Abbate, F. Castiglione, F. Lebon, G. Longhi, A. Longo, A. Mele, W. Panzeri, A. Ruggirello and V. T. Liveri, J. Phys. Chem. B, 2009, 113, 3024-3033.

30 S. Abbate, A. Ciogli, S. Fioravanti, F. Gasparrini, G. Longhi, L. Pellacani, E. Rizzato, D. Spinelli and P. A. Tardella, Eur. J. Inorg. Chem., 2010, 6193-6199.

31 P. Scafato, F. Caprioli, L. Pisani, D. Padula, F. Santoro, G. Mazzeo, S. Abbate, F. Lebon and G. Longhi, Tetrahedron, 2013, 69, 10752-10762.

32 S. Grimme, J. Antony, S. Ehrlich and H. Krieg, J. Chem. Phys., 2010, 132, 184103.

33 S. Grimme, J. Comput. Chem., 2006, 27, 1787-1799.

34 A. Schäfer, H. Horn and R. Ahlrichs, J. Chem. Phys., 1992, 97, 2571-2577.

35 G. Longhi, S. Abbate, P. Scafato and C. Rosini, Phys. Chem. Chem. Phys., 2010, 12, 4725-4732.

36 M. Passarello, S. Abbate, G. Longhi, S. Lepri, R. Ruzziconi and V. P. Nicu, J. Phys. Chem. A, 2014, 118, 4339-4350. 